ORIGINAL ARTICLE

\title{
Molecular genetic analysis of VRK1 in mammary epithelial cells: depletion slows proliferation in vitro and tumor growth and metastasis in vivo
}

TP Molitor and P Traktman

The vaccinia-related kinases (VRKs) comprise a branch of the casein kinase family. VRK1, a ser/thr kinase with a nuclear localization, is the most well-studied paralog and has been described as a proproliferative protein. In lower eukaryotes, a loss of VRK1 activity is associated with severe mitotic and meiotic defects. Mice that are hypomorphic for VRK1 expression are infertile, and depletion of VRK1 in tissue culture cells can impair cell proliferation and alter several signaling pathways. VRK1 has been implicated as part of a 'gene-expression signature' whose overexpression correlates with poor clinical outcome in breast cancer patients. We present here our investigation of the role of VRK1 in the growth of normal (MCF10) and malignant (MDA-MB-231) human mammary epithelial cells, and demonstrate that shRNA-mediated depletion of VRK1 slows their proliferation significantly. Conversely, stable overexpression of a FLAG-tagged VRK1 transgene imparts a survival advantage to highly malignant MDA-MB-231 cells under conditions of nutrient and growth factor deprivation. Moreover, in a murine orthotopic xenograft model of breast cancer, we demonstrate that tumors depleted of VRK1 show a 50\% reduction in size from 4-13 weeks postengraftment. The incidence and burden of distal metastases in the lungs and brain was also significantly reduced in mice engrafted with VRK1-depleted cells. These studies demonstrate that VRK1 depletion or overexpression has an impact on the proliferation and survival of cell lines derived from normal or malignant mammary tissue, and moreover show that depletion of VRK1 in MDA-MB-231 cells reduces their oncogenic and metastatic properties in vivo.

Oncogenesis (2013) 2, e48; doi:10.1038/oncsis.2013.11; published online 3 June 2013

Subject Categories: molecular oncology

Keywords: protein kinase; VRK1; breast cancer; tumorigenesis; cell proliferation; orthotopic xenograft

\section{INTRODUCTION}

Reversible protein phosphorylation is arguably the most important regulator of dynamic cellular processes. Cellular proliferation, and in particular cell cycle progression, exemplifies the power of phosphorylation cascades mediated by a plethora of cellular kinases and phosphatases. Deregulation of the cell cycle and its intrinsic checkpoints is a significant contributor to the development and progression of cancer. ${ }^{1}$ The vaccinia-related kinases (VRKs) comprise a branch of the casein kinase family. No homologs are found in yeast, but a single, essential VRK gene is encoded by $D$. melanogaster and C. elegans and three paralogs are found in mammals: VRK1 (nuclear), VRK2 (ER and nuclear membranes) and VRK3 (nuclear, inactive pseudokinase). ${ }^{2}$ VRK1 is correlated with a proliferative phenotype ${ }^{3,4}$ and its overexpression has been associated with several types of cancer. ${ }^{3,5-7}$

VRK1 can phosphorylate and regulate the CRE binding protein, enhancing cyclin D expression. ${ }^{8}$ VRK1 is transcriptionally downregulated following serum starvation, and the kinetics of its accumulation after serum restimulation matches those of c-Myc and c-Fos. ${ }^{9}$ Transient depletion of VRK1 in primary fibroblasts has been shown to reduce cyclin D1 and phospho-Rb levels, with a concomitant reduction in cellular proliferation. ${ }^{9}$ Our laboratory has reported that male mice with hypomorphic expression of VRK1 are infertile due to a loss of proliferating spermatogonia, ${ }^{10}$ which has been confirmed by others. ${ }^{11,12}$ VRK1-mediated phosphorylation of $\mathrm{BAF}$, which associates with both DNA and nuclear envelope constituents and is essential for proper nuclear envelope breakdown and assembly, ${ }^{13}$ has been observed in both C. elegans and mammalian cells. ${ }^{13,14}$ VRK1-mediated phosphorylation of histone $\mathrm{H} 3$ is postulated to affect chromosome condensation during mitosis. ${ }^{15}$ The dynamic phosphorylation of these proteins by VRK1 supports the hypothesis that VRK1 may regulate events at both $\mathrm{G}_{0} / \mathrm{G}_{1}$ and $\mathrm{G}_{2} / \mathrm{M}$.

The association of disregulated VRK1 expression with breast cancer emerged from two complementary lines of research. ${ }^{16,17}$ Genes whose expression was attenuated as mammary epithelial cells differentiated into mature acini in 3D cultures were identified by microarray analysis. Repression of such genes was deemed likely to be important during mammary duct homeostasis, and conversely, disregulation of such genes was thought likely to be linked to oncogenesis. VRK1 was among 22 such genes. ${ }^{16}$ Second, the results of this microarray analysis were compared with those of breast cancer biopsies: VRK1 was identified as one of 19 genes whose overexpression correlated with poor prognostic outcome in breast cancer patients. ${ }^{16}$ In a follow-up study, the same 19-gene signature was used to predict clinical outcome within large sets of annotated microarray data. ${ }^{17}$ A statistically significant correlation of VRK1 overexpression with poor clinical outcome was confirmed. 
Herein, we examine the role of VRK1 in regulating the growth of mammary epithelial cells both in vitro and in vivo. In vitro, we demonstrate that stable depletion of VRK1 in both non-transformed MCF10a cells and highly transformed and metastatic MDA-MB-231 slows cell proliferation. In contrast, overexpression of a VRK1 transgene enhanced the ability of MDA-MB-231 cells to survive and proliferate under low-serum conditions. In vivo, we assessed the contribution of VRK1 to MDA-MB-231-mediated mammary tumor formation in mice. Tumors stably depleted of VRK1 grew more slowly and exhibited a diminished incidence of metastasis. These are the first studies to investigate the contribution of VRK1 to mammary epithelial cell proliferation in vitro and to directly test the effect of VRK1 expression on tumor growth and metastasis in vivo.

\section{RESULTS}

VRK1 depletion slows the apparent doubling time of MCF10a and MDA-MB-231 cells.

High levels of VRK1 expression have been associated with proliferative cell types and a variety of cancers, and several lines of study have implied a role for VRK1 in cell cycle progression. $^{8,14,15,18}$ VRK1 depletion has been shown to compromise the survival and proliferation of HCT116 lung carcinoma cells, and to increase the doubling time of non-transformed WS1 fibroblasts from $\sim 42$ to $\sim 60 \mathrm{~h} .{ }^{9}$ As VRK1 overexpression has been associated with poor clinical outcome in breast cancer patients, we focused on normal (MCF10a) and malignant (MDA-MB-231) mammary epithelial cell lines to assess
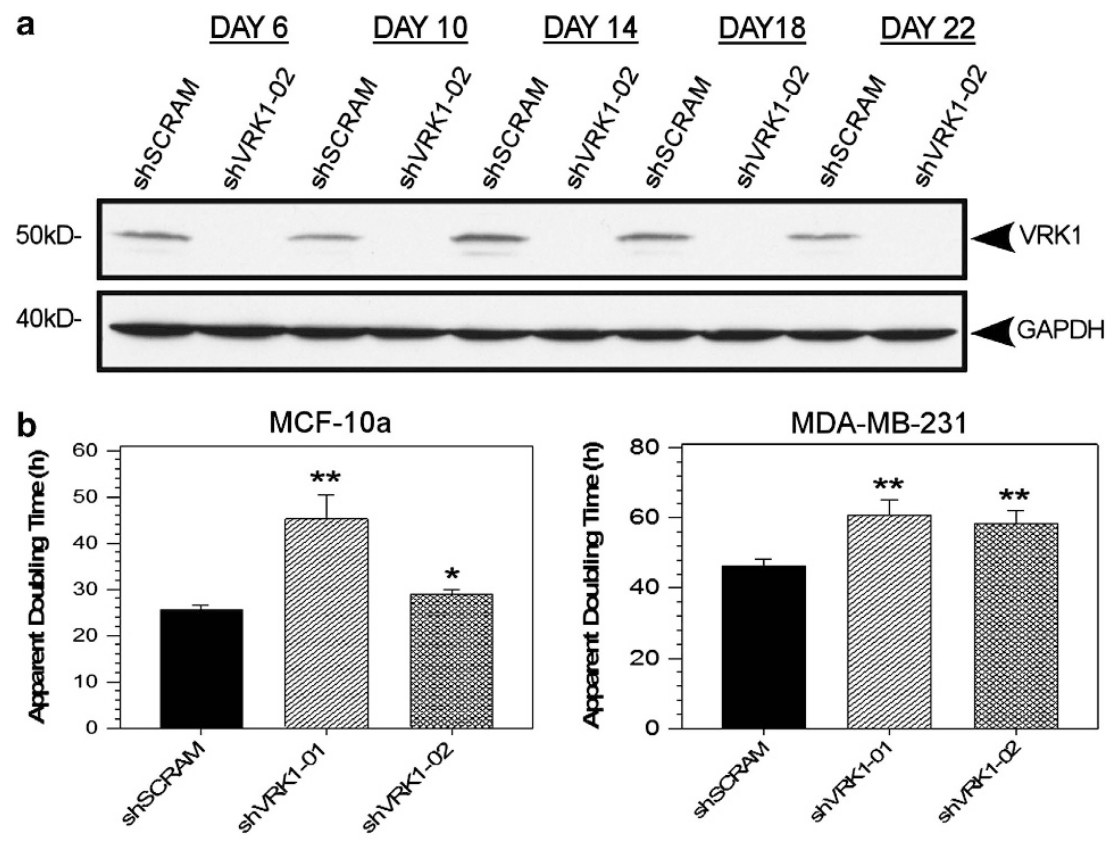

C
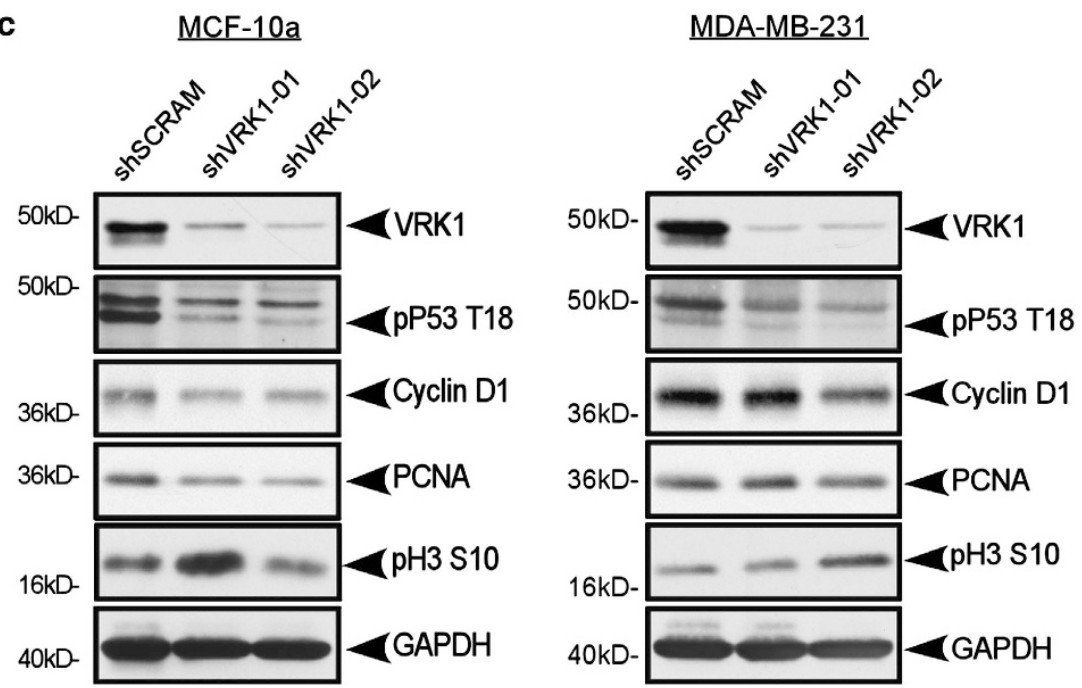

Figure 1. Stable shRNA-mediated depletion of VRK slows the proliferation of MCF10a and MDA-MB-231 cells. (a) MDA-MB-231 cells were stably depleted of VRK1 by lentiviral delivery of shRNA and selection for Puro ${ }^{R}$. Cell lysates were prepared at multiple time points following infection and VRK1 levels were assessed by immunoblot analysis. VRK1 expression in control (shSCRAM) cells and cells expressing shVRK1-02 were averaged over time and the knockdown of VRK1 was calculated to be $96 \pm 0.59 \%$. (b) MCF10a, and MDA-MB-231 cells were engineered to express two unique VRK1-directed shRNAs (shVRK1-01, shVRK1-02) or a control shRNA (shSCRAM). Puro populations were seeded at equal densities and incubated under normal growth conditions for $72-144 \mathrm{~h}$ (cell type dependent, $n>8$ ). Cell number and viability were determined by flow cytometry and apparent doubling time was calculated. Depletion of VRK1 leads to a significant increase in apparent doubling time in both MCF10a and MDA-MB-231 cells. $\left.{ }^{*} P<0.05,{ }^{* * P}<0.01\right)$. (c) Immunoblot analysis was used to confirm the depletion of VRK1 as appropriate $(n>8)$ and to monitor the expression of proteins diagnostic for different stages of the cell cycle. 
how VRK1 would contribute to their survival, proliferation and cell cycle progression.

We utilized lentiviral transduction to engineer pure populations of Puro $^{R}$ cells stably expressing VRK1-directed (shVRK1-01, shVRK1-02) or control (shSCRAM) shRNAs. VRK1 depletion was highly efficient (knockdown $=96.3 \% \pm 0.59, n=7$ ) and sustained for $>20$ days (Figure 1a). Equal numbers of viable cells were seeded and the apparent doubling time was calculated over several passages. We observed a statistically significant increase in doubling time for all cell types upon depletion of VRK1 (Figure 1b). The doubling time of the control MCF10a cells was $25.5 \pm 1.32 \mathrm{~h}$, and was increased to $45.2 \pm 5.32 \mathrm{~h}$ by shVRK1-01 expression and $28.2 \pm 1.21 \mathrm{~h}$ by shVRK1-02 expression. (In some experiments, shVRK1-02 was less effective at depleting VRK1 in this cell type, which may explain the more modest impact seen on the apparent doubling time). In the MDA-MB-231 cell line, depletion of VRK1 via the VRK1-01 and VRK1-02 targeting sequences were equally effective in inflating the doubling time from the control value of $45.6 \pm 1.93 \mathrm{~h}$ to $57.0 \pm 4.64 \mathrm{~h}$ and $57.4 \pm 3.5 \mathrm{~h}$, respectively.

From these data we conclude that both non-transformed and transformed mammary epithelial cells exhibit a slow-growth phenotype upon ablation of VRK1. Previously, the early S-phase delay seen upon VRK1 depletion in primary cells has been ascribed in part to a reduction in cyclin D1 expression. ${ }^{8,9}$ Representative immunoblots from our growth curve experiments are shown in Figure 1c. MCF10a cells depleted of VRK1 display a very modest reduction in the levels of cyclin D1 and PCNA, suggesting a possible impairment in the G1/S transition. However, expression of the mitotic marker phospho-H3 Ser10 was either stable or elevated upon VRK1 depletion, indicating that transit into mitosis was not compromised. Immunoblot analysis of the MDA-MB-231 cells depleted of VRK1 did not reveal any discernible impact on the accumulation of representative cell cycle specific proteins. In both cell types, VRK1 depletion did reduce the levels of phospho-p53 Thr18; this posttranslational modification by VRK1 has been previously reported. ${ }^{19,20}$

The simplest interpretation of these findings is that VRK1 depletion slows the cell cycle progression of all cells in the population. Although the calculation of 'net proliferation' could be affected by an increased number of dying cells, our flow cytometric live/dead analyses were not consistent with this possibility. It is also possible that VRK1 depletion increased the number of quiescent cells in the population.

VRK1 depletion alters the cell cycle distribution of cells in the G1 and $\mathrm{S}$ phases in MCF-10a, but not MDA-MB-231 cells

Previously it was demonstrated by immunofluorescence that transient depletion of VRK1 in HeLa cells diminished the fraction a
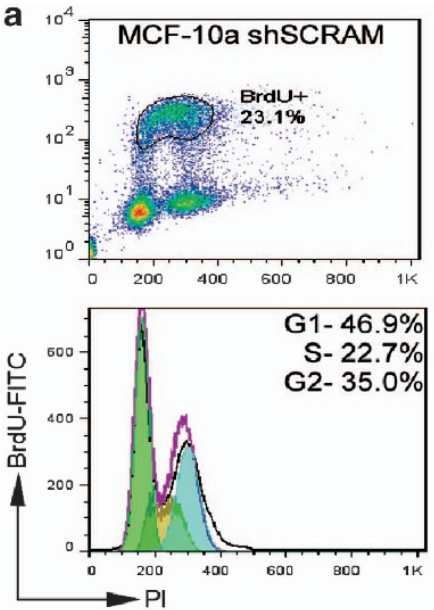

b 1
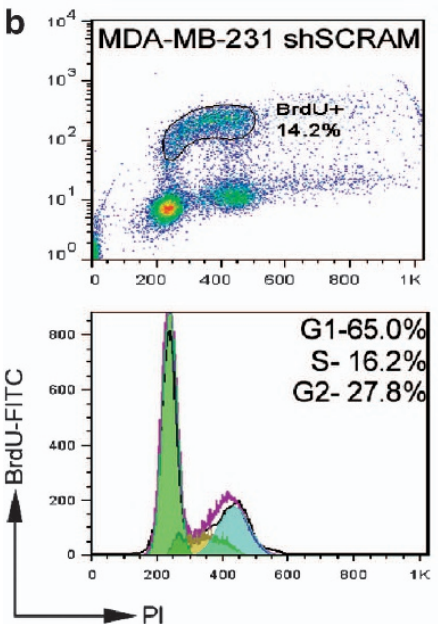
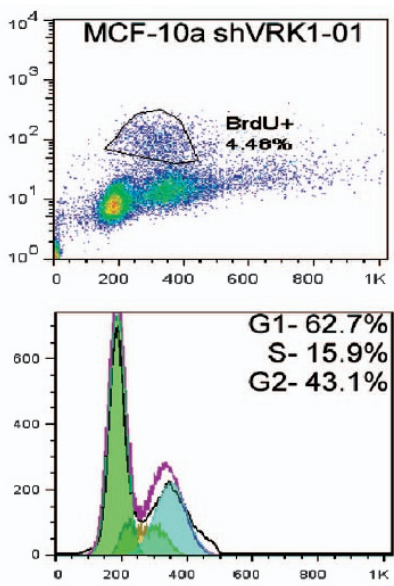

S- $15.9 \%$
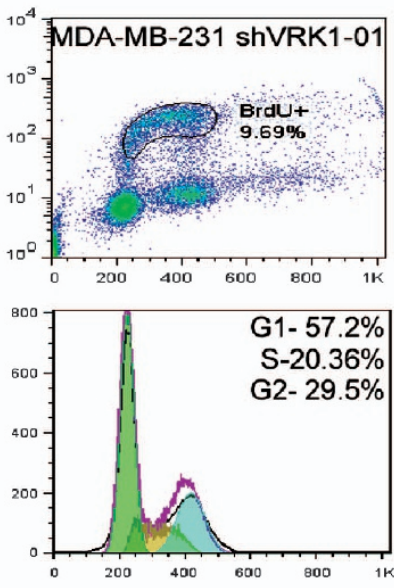
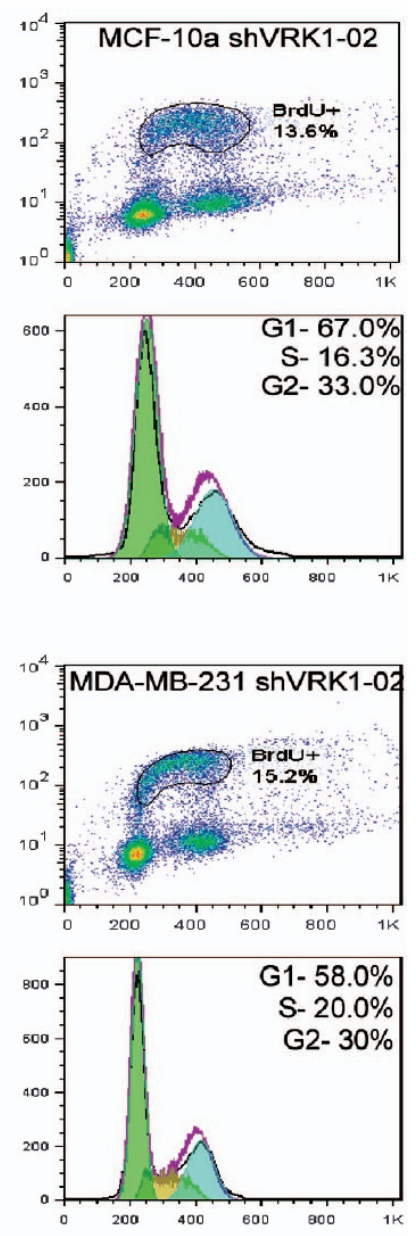

Figure 2. Depletion of VRK1 in MCF10a, but not MDA-MB-231, increases the $\mathrm{G} 1$ and reduces the $\mathrm{S}$ phase population. (a, b) Cell cycle progression was assessed by a short $(60 \mathrm{~min})$ pulse of BrdU, followed by labeling with a FITC conjugated anti-BrdU- antibody to detect DNAreplicating cells, combined with propidium iodide staining as a measure of DNA content. Flow cytometry was performed on MCF10a (a) and MDA-MB-231 (b) VRK1-depleted (shVRK1-01,-02) and control (shSCRAM) cells. The percentage of BrdU + cells is indicated by the gated population in the top panels. DNA content was analysed using the Watson-Pragmatic cell cycle method and percentage values for G0/G1 ( $2 \mathrm{~N}$ DNA content), S (intermediate DNA content) and G2/M (4 N DNA content) cells are shown in the bottom panels. 
of cells undergoing DNA replication. ${ }^{8}$ We took a similar approach to assess the impact of VRK1 depletion on S phase by monitoring the fraction of cells incorporating BrdU into nascent DNA during a 1-h pulse. VRK1-depleted and control cells were stained with an anti-BrdU antibody as well as PI to identify cells within distinct stages of the chromosome cycle. Depletion of VRK1 within MCF10a cells (Figure 2a) diminished the number of cells actively undergoing DNA synthesis from $23.1 \%$ (shSCRAM) to 4.48 or $13.6 \%$ (shVRK-01 and -02, respectively) (top panels). The PI analysis (bottom panels) indicates that this reduction reflects a 'shift' of cells into G1; whereas the fraction of cells in $\mathrm{G} 1$ is $46.9 \%$ in the shSCRAM population, it increased to 62.7 and $67 \%$ in cells expressing shVRK1-01 or -02, respectively. (A modest increase in the G2/M population is seen in the shVRK1-01 population consistent with the increase in phospho-H3 Ser10 signal shown in Figure 1c.) These data strongly support a role for VRK1 in G1 progression and/or passage through the G1/S checkpoint in MCF-10a cells. This G1 delay is likely to be the major determinant of the slower doubling time seen for the VRK1-depleted MCF10a cells (Figure 1).

A different profile was seen in the MDA-MB-231 cell line (Figure $2 \mathrm{~b}$ ). The proportion of BrdU + cells, which was $14.2 \%$ of the shSCRAM population, was largely unaffected by expression of either shVRK1-01 or -02 (9.69\% or $15.2 \%$, respectively). Moreover, the $\mathrm{PI}$ analysis indicated that the overall cell cycle distribution of the population was unaffected by VRK1 depletion (Figure $2 \mathrm{~b}$ bottom panels). The $\mathrm{G} 1$ phase of the MDA-MB-231 cells is already quite long, with $65 \%$ of the control cells residing therein; hence, something other than VRK1 may be rate-limiting in the passage of MDA-MB-231 cells into S phase. Although VRK1 depletion causes a highly significant increase in the apparent doubling time of the MDA-MB-231 cells (Figure 1), the effect does not appear to be limited to a particular phase of the cell cycle.

Overexpression of exogenous VRK1 does not affect proliferation under normal culture conditions

As VRK1 overexpression is correlated with poor clinical prognosis in breast cancer patients, we wanted to examine the impact of stable overexpression of VRK1 on MCF-10a and MDA-MB-231 proliferation. Cell populations stably expressing $3 \times$ FLAG-VRK1 under the regulation of a CMV promoter were generated; overexpression was calculated to be 6.87-fold and 6.19-fold above endogenous levels in MCF10a-VRK1 and MDA-MB-231-VRK1 cells, respectively (Figure $3 \mathrm{a}$ ). Under normal culture conditions, no significant change in the apparent doubling time of the cells was seen (Figure 3b), nor was an impact on cyclin D1 expression. Our flow cytometric method for cell counting provides quantification of both the total and viable cell numbers, and thus we could also conclude that the levels of cell death were not altered by VRK1 overexpression. The increased levels of phosphorylated BAF, a validated and highly robust substrate for VRK1, ${ }^{14}$ provided evidence that the overexpressed and epitope-tagged VRK1 was indeed functional.
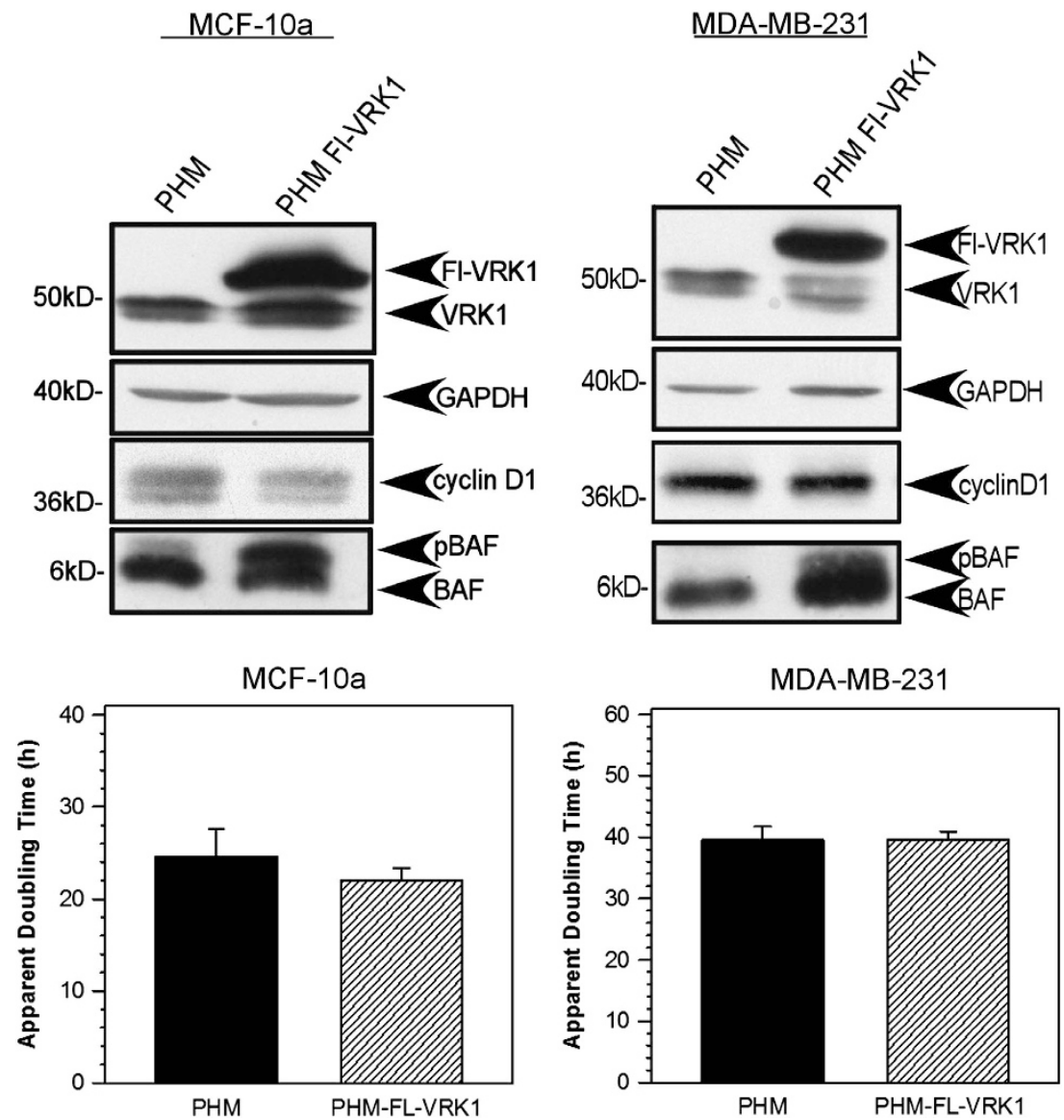

Figure 3. VRK1 overexpression does not affect the proliferation of MCF-10a or MDA-MB-231 cells under normal growth conditions. (a) MCF10a and MDA-MB-231 cells were engineered to overexpress a $3 \times$-FLAG-epitope tagged version of VRK1 under the control of the CMV promoter (PHM-FI-VRK1) along with a HYGROR ${ }^{R}$ cassette. Protein expression was assessed by immunoblot analysis; the FL-VRK1 transgene was calculated to be expressed at levels sixfold greater than enodogenous levels. (b) HYGRO ${ }^{R}$ populations were seeded at equal densities and incubated under normal growth conditions for $72-144 \mathrm{~h}(n=4)$. Cell number and viability were determined by flow cytometry and the apparent doubling time was calculated. 
Overexpression of VRK1 stimulates MDA-MB-231 survival and growth under serum-starved conditions.

We postulated that VRK1 overexpression might augment the proliferation of cells confronting nutrient-limiting conditions, as might be encountered in vivo during tumorigenesis. Neither control (PHM) or VRK1-overexpressing MCF10a cells could proliferate in the presence of $0.2 \%$ serum (Figure $4 a$, top panels). After 7 days, both populations had dwindled in number although the MCF10a-VRK1 cells had a slight survival advantage. However, when MDA-MB-231 cells were subjected to serum starvation, VRK1 overexpression had a clear effect on cell survival and growth (Figure $4 \mathrm{a}$, bottom panels). There was a significant increase in the number of viable cells in the 231-PHM-VRK1 populations after 7 days of serum starvation compared with the 231-PHM control population (Figure $4 b$ ).

To determine whether VRK1 overexpression might be diminishing a possible apoptotic response elicited by serum starvation, a luminescence-based assay for activated caspase 3/7 was performed on culture supernatants. As a control for the assay, media were collected from MDA-MB-231 cells cultured for $24 \mathrm{~h}$ under normal culture conditions in the absence or presence of staurosporine, a potent inducer of apoptosis (Figure 4c, left two bars); a dramatic difference was readily observed. Media collected from control (PHM) or VRK1-overexpressing (VRK1) cells after 7 days of serum starvation were similarly assayed. There was a statistically significant reduction (15\%) in caspase $3 / 7$ activity in the spent media harvested from the VRK1-overexpressing cells relative to control cells (Figure $4 \mathrm{c}$ ). These data demonstrate that there is an apoptotic response in the serum-starved MDA-MB-231 cultures that is partially overcome by VRK1 overexpression.

Immunoblot analysis (Figure 4d) showed that expression of the G1/S proteins cyclin D1 and PCNA were nearly equivalent in the 231-PHM control and VRK1-overexpressing cells under normal growth ( + serum) conditions or after 3 days of serum starvation. MDA-MB-231 cells express high levels of mutant p53, which dampens the G1/S checkpoint even in the absence of nutrients, oxygen or in this case serum. ${ }^{22}$ After 7 days in $0.2 \%$ serum, both cell types showed a comparable reduction in cyclin D1 levels a

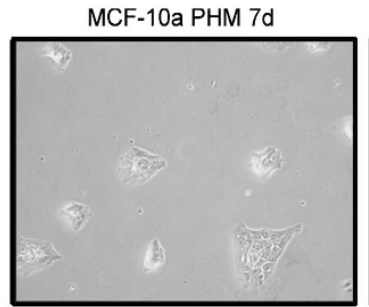

231- PHM 7d

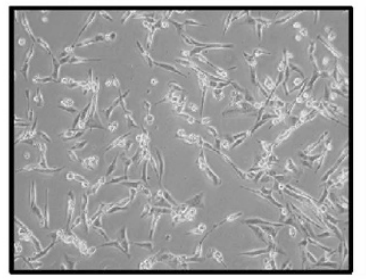

C

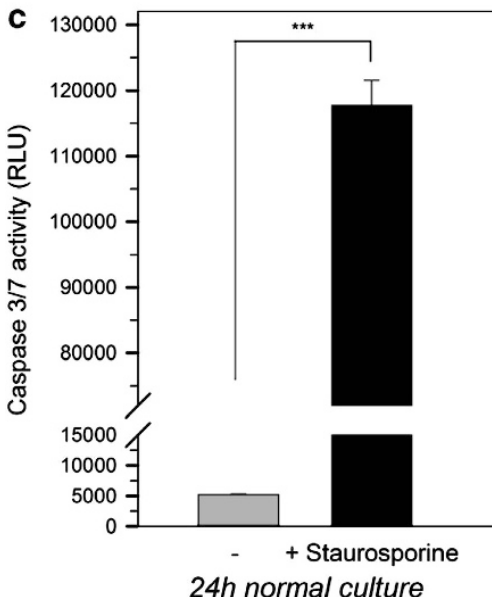

$24 \mathrm{~h}$ normal culture
MCF-10a PHM-VRK1 7d

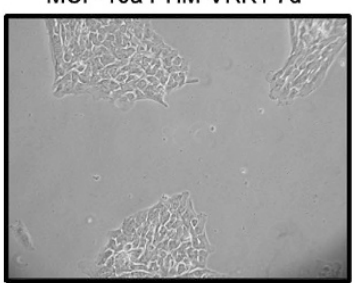

231 PHM-VRK1 7d

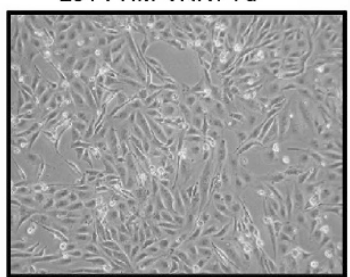

b

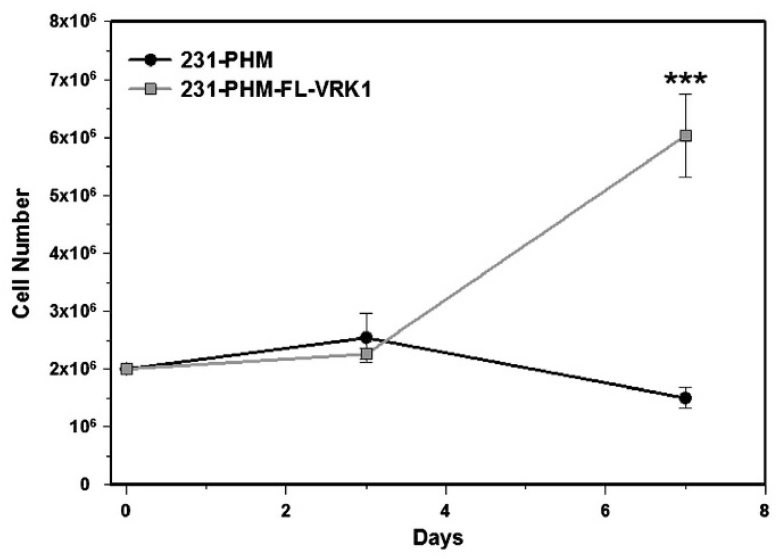

d

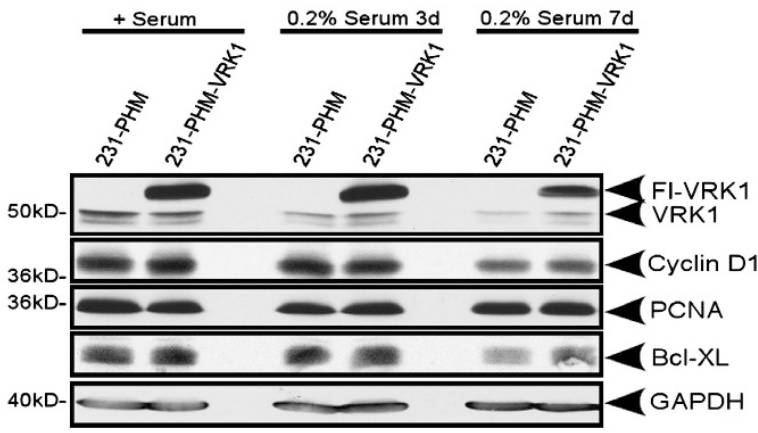

Figure 4. VRK1-overexpressing cells have a growth advantage under conditions of nutrient and growth factor deprivation. (a) Equal numbers of control (PHM) and VRK1-overexpressing (PHM-VRK1) cells (both MCF10a and MDA-MB-231) were cultured in $0.2 \%$ serum and in the absence of any growth factor supplementation for 7 days. Representative phase images documenting the observed cell densities are shown for each population. (b) Equal numbers of MDA-MB-231 PHM and PHM-VRK1 cells were cultured in 0.2\% serum and in the absence of any growth factor stimulation for 3 to 7 days. Surviving cells were counted from each population and displayed graphically over time $(n=5)$. (c) Left bars: culture media harvested from MDA-MB-231 cells cultured for $24 \mathrm{~h}$ hours in the absence $(-)$ or presence $(+)$ of staurosporine were assayed for caspase 3/7 levels; the luminescent readout is reported as RLU. Right bars: culture media were also harvested from MDA-MB-231 PHM and MDA-MB-231-PHM-VRK1 cultures maintained for 7 days under conditions of serum starvation. In each case, two biological replicates were performed and supernatants were assayed in duplicate at one time. (d) 231-PHM and 231-PHM-VRK1 populations were cultured under normal growth conditions ( + serum) or serum-starved conditions $(0.2 \%$ serum) for 3 and 7 days, and cell lysates were prepared and subjected to immunoblot analysis $(* * * P<0.001)$. 
(231-PHM, 0.69-fold reduction; 231-PHM-VRK1, 0.64-fold reduction), while PCNA levels persisted. 231-PHM-VRK1 cells had a twofold increase in the levels of the prosurvival $\mathrm{BCl}-\mathrm{XL}$ protein relative to the control 231-PHM cells. It is likely that increased survival signaling and reduced apoptosis contribute to the growth advantage observed in VRK1 overexpressing cells cultured under low-serum conditions. Following 7 days of culture in low serum, the level of the endogenous VRK1 protein was reduced in both cell lines, although the exogenous 3XFLAG-VRK1 protein was maintained at high levels in the 231-PHM-VRK1 cells. The CMV promoter driving transgene expression is likely to be insensitive to serum starvation, and the constitutive VRK1 expression that results may alter the cell's natural response to nutrient limitation, promoting survival and proliferation.
VRK1-overexpressing MDA-MB-231 cells do not exit the cell cycle after prolonged serum starvation

The data presented above (Figure $4 \mathrm{~b}$ ) indicated that the 231-PHM cells were not proliferating during prolonged culture in low-serum medium despite the presence of cyclin D1 and PCNA. To gain additional insight into whether a subset of cells were proliferating while the majority were not, we utilized flow cytometry to analyze the cell cycle distribution. Under normal growth conditions ( + serum), VRK1 overexpression did not increase the proportion of cells in $\mathrm{S}$ phase or alter the cell cycle distribution of MDA-MB-231 cells (Figures $5 \mathrm{a}$ and c, left panels). However, following 3 days of culture in $0.2 \%$ serum, the percentage of BrdU + cells was somewhat higher in the 231-PHM-VRK1 cell line than the control (Figure 5a, middle panels), and the average
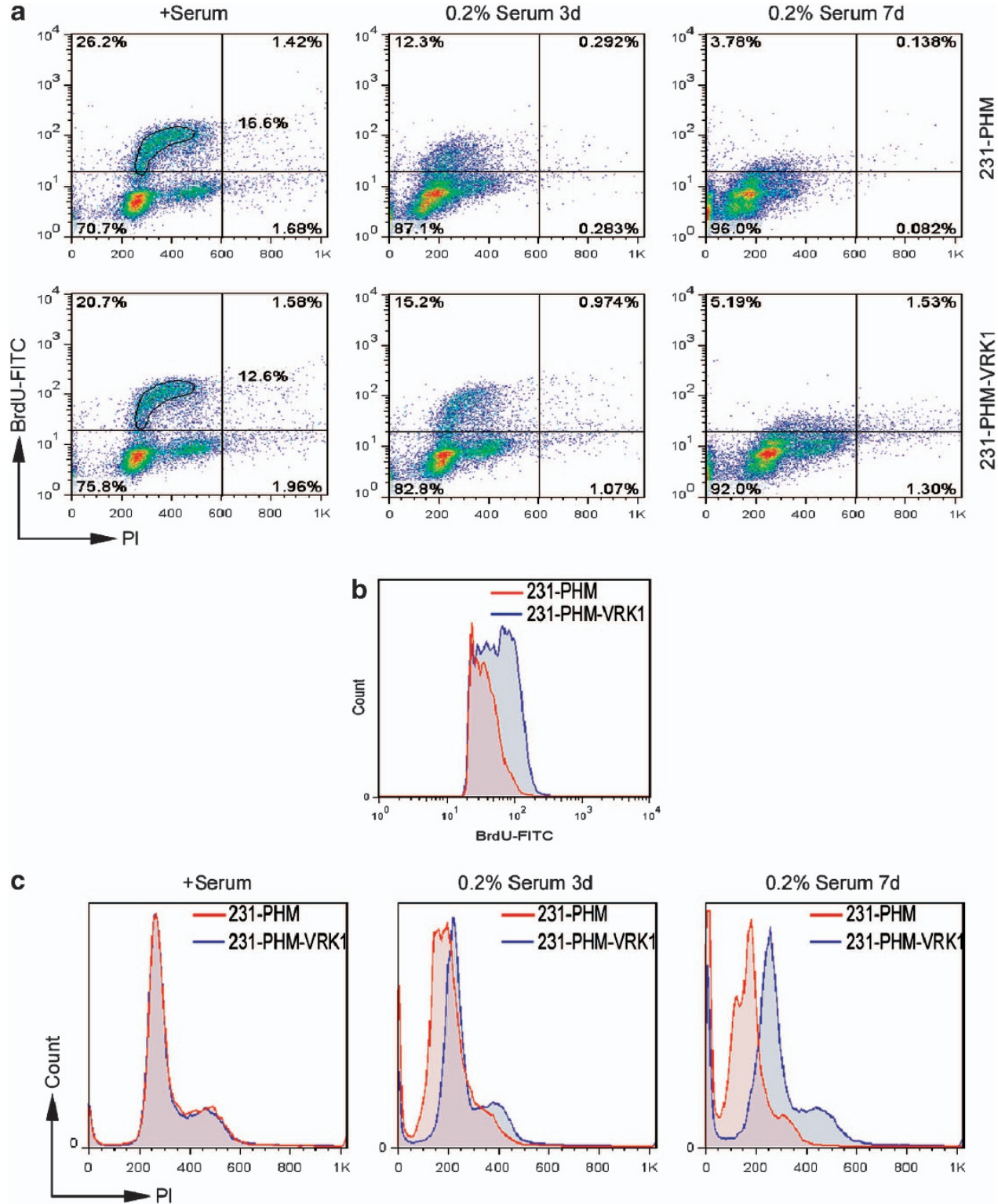

Figure 5. Overexpression of VRK1 in serum-starved MDA-MB-231 cells lessens cell cycle arrest and prevents DNA hypercompaction. (a) Cell cycle progression was assessed by a short $(60 \mathrm{~min})$ pulse of BrdU, followed by labeling with a FITC conjugated anti-BrdU antibody and propidium iodide staining. Flow cytometry was performed on MDA-MB-231 VRK1-overexpressing (231-PHM-VRK1) cells and control (231-PHM) cells under normal growth conditions ( + serum) or 3 and 7 days of low-serum growth conditions (0.2\%). (b) Overlay of cells from the BrdU positive gate (quadrant I) after 3 days of growth under low-serum conditions. VRK1-overexpressing cells (blue line) have a larger proportion of cells actively synthesizing DNA than controls (red line). (c) PI staining of each population reveals a marked leftward shift in the G1 peak of 231 PHM cells following serum starvation and loss of the G2/M population. 231-FL-VRK1 cells maintain the normal position of the G1 peak, as well as a small population of G2/M cells following 7 days of growth in low serum. 
intensity of staining within this population was clearly greater (Figure 5b). Additionally, nearly all of the control 231-PHM cells had accumulated in G1/S, while the 231-PHM-VRK1 line still displayed a G2/M population. Both populations appear to have halted DNA synthesis after 7 days of serum starvation, but the VRK1-overexpressing line maintained a higher number of cells in G2/M. A subG1 population of cells, indicative of apoptosis, can be seen to increase in both cell lines during serum starvation (mean fluorescence intensity of $0-50$, along the y-axis).

The most striking result can be seen from careful analysis of the $\mathrm{PI}$ distribution. The mean fluorescence intensity of the G1 peak in the control cells (red) shifts to the left from 250-300 in normal medium to $150-200$ in the serum-starved cells. This leftward shift, which reflects diminished PI binding, is indicative of DNA hypercompaction (or reduced DNA content). These data suggest that the majority of the nutrient-starved 231-PHM cells may be exiting the cell cycle and entering a G0-like quiescent state, a capability that they retain despite their highly transformed state. ${ }^{21,23}$ In contrast, the mean fluorescence intensity of the G1 peak in the VRK1-overexpressing cells does not shift to the left, even after 7 days of serum starvation. Thus, although few cells are in $S$ phase, the cells have not withdrawn from the cell cycle. The differential response to nutrient withdrawal evoked by VRK1 overexpression is provocative given the association of VRK1 overexpression with oncogenesis, as nutrient stress is often encountered within the primary tumor environment before metastasis. $^{24}$

VRK1 depletion in MDA-MB-231 cells slows tumor growth in a murine orthotopic xenograft model of breast cancer

To move beyond correlative data linking VRK1 to cancer, we investigated whether VRK1 depletion would impact tumorigenesis. Using MDA-MB-231 cells that had been passaged through mice to enhance their tumorigenicity and engineered to express luciferase, allowing tumor growth to be tracked non-invasively using biophotonic imaging, ${ }^{25}$ we established lines expressing shSCRAM or shVRK1-02. An engraftment was performed by injecting $1.5 \times 10^{6}$ cells into the 4th mammary fatpad of immune-deficient hairless-SCID mice. Tumor progression was monitored weekly by bioluminescent imaging and caliper measurement. Displayed in Figure $6 a$ are representative heat maps of the luciferase signal recorded following bioluminescent imaging. At the time of engraftment (0 weeks) 231-Luc shSCRAM and -shVRK1-02 tumors displayed equal levels of luminescence (RLU). By 6 weeks it was readily discernible that the 231-LucshVRK-02 tumors were smaller than the control tumors (shSCRAM). This disparity was more apparent at 10 weeks, but by this time tumor necrosis and ulceration were evident in both experimental sets of mice (Figure 6a, cool heat map in necrotic center). These changes in tumor status are problematic as they quench the luminescent signal; caliper measurement becomes a more reliable means of assessing tumor size at this point (Figure 6c). Quantification of tumor progression as assessed by biophotonic imaging for the initial 9 weeks is displayed graphically in Figure $6 \mathrm{~b}$ ( $n=13$ shSCRAM, $n=15$ shVRK1-02). A significant difference in tumor volume between the control and VRK1-depleted tumors is seen as early as 4 weeks postengraftment. Two animals bearing 231-Luc shSCRAM tumors succumbed to their tumor burden at weeks 5 and $6(\dagger)$. None of the mice bearing VRK1-depleted tumors died from their tumor burden, and one mouse was cleared of its engraftment by week 4 . At 8 weeks postengraftment, VRK1-depleted tumors were reduced in volume by an average of $\sim 57 \%$ as assessed by RLU monitoring, and by an average of $\sim 50 \%$ as assessed by caliper measurement (Figure 6c). A statistically significant difference in tumor size was maintained throughout the rest of the 12-week experiment. These data indicate that depletion of VRK1 in the highly malignant MDA-MB-231 cells can retard the rate of tumor growth by an average of $50 \%$ following orthotopic engraftment. (Post-hoc immunoblot analysis of tumor extracts confirmed that VRK1 a
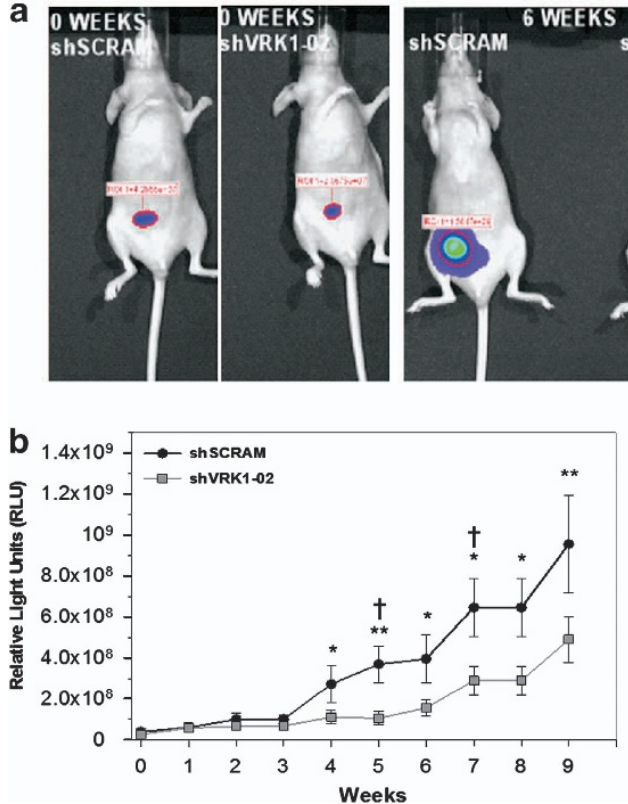
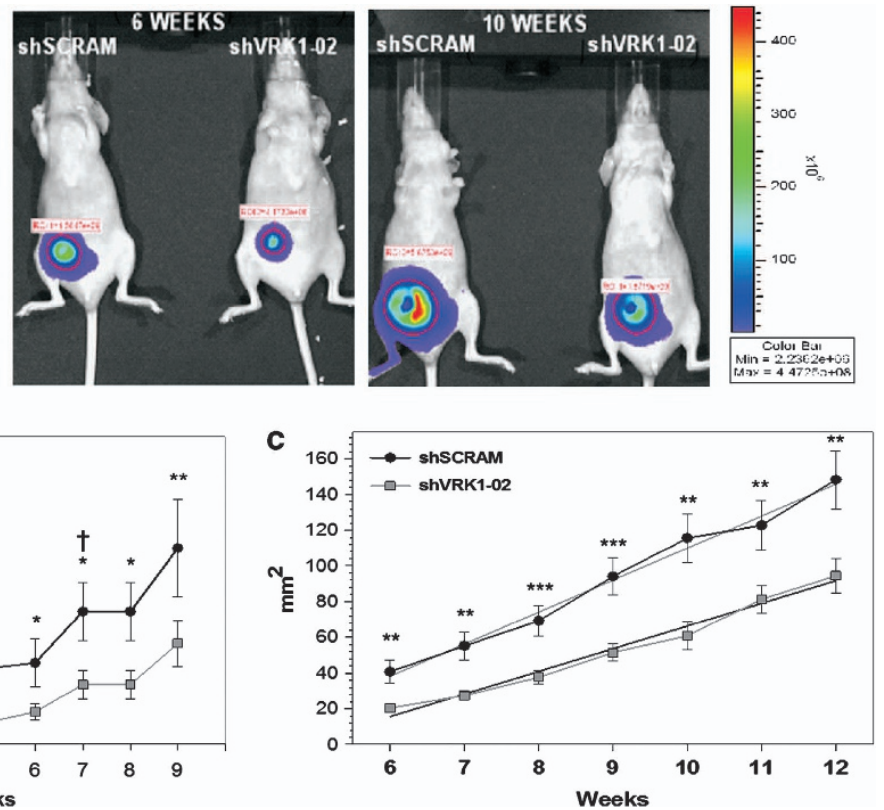

Figure 6. Depletion of VRK1 in MDA-MB-231 reduces tumor size in a orthotopic xenograft model of breast cancer. SCID hairless outbred mice were orthotopically engrafted with $1.5 \times 10^{6}$ MDA-MB-231 LUC cells stably expressing luciferase and either a control (shSCRAM) or VRK1targeting (shVRK1-02) shRNA. (a) Representative bioluminescent images showing equal engraftment at time zero (0 weeks), but reduced tumor size for shVRK1-02-expressing cells after 10 weeks. (b) Mean tumor burden is expressed as a measure of luciferase activity (RLU) over 9 weeks ( $n=13$ shSCRAM, $n=15$ shVRK1-02). Two mice engrafted with control, shSCRAM-expressing cells succumbed to their tumor burden ( $\dagger$ ). (c) Tumor size was assessed by caliper measurement and reported as mean tumor area from 4 to 12 weeks. Statistical significance was determined by one-way ANOVA (ANOVA: $P<0.0001, F=9.09$ ) and Dunn's post-hoc analysis. The slope of the linear regression lines representing control and VRK1-depleted tumor growth were significantly different $\left({ }^{*} P<0.05,{ }^{* *} P<0.01,{ }^{* * *} P<0.001\right)$. 
a
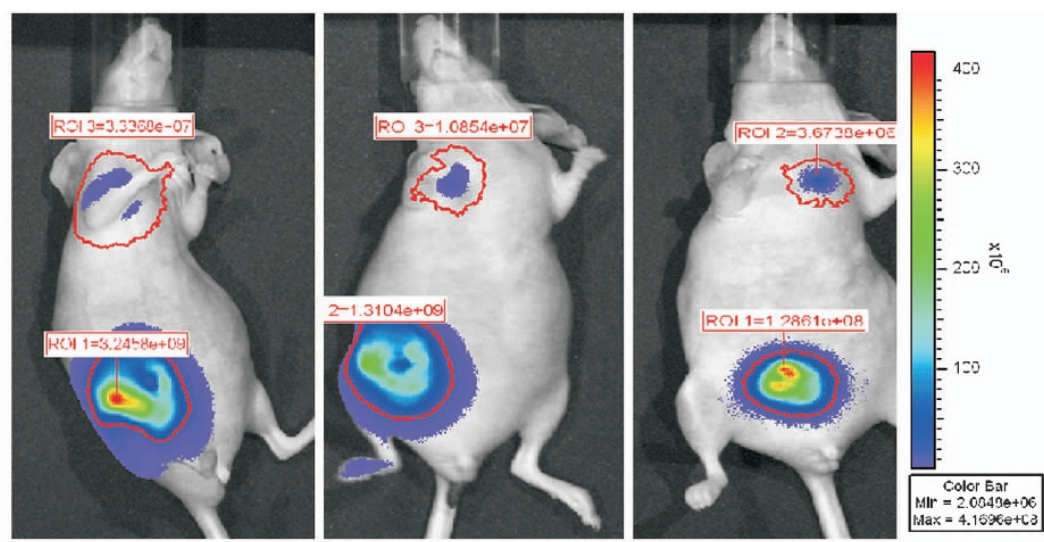

b
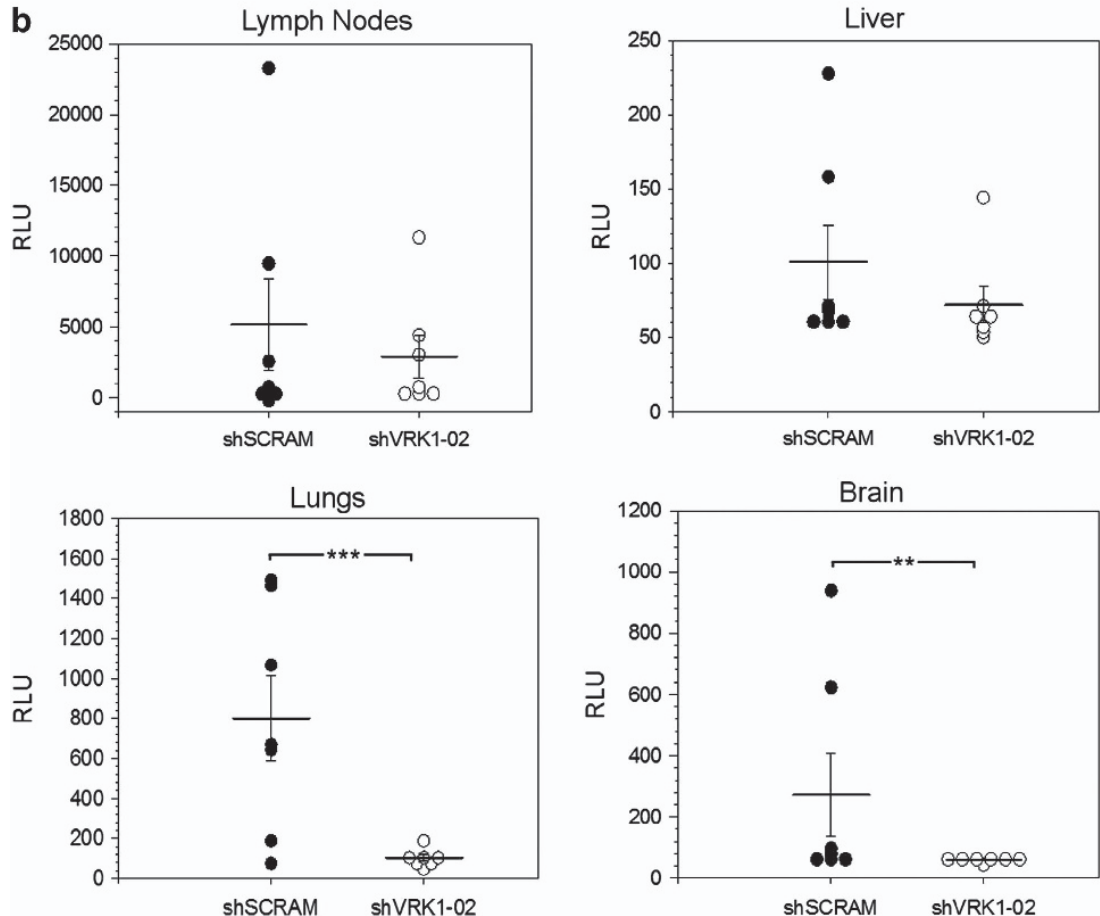

Figure 7. Mice bearing VRK1-depleted tumors have a reduced incidence and burden of metastasis to the lungs and brain. (a) Visual recognition of lung metastases in three individual shSCRAM 231-Luc tumor bearing mice via biophotonic imaging. No lung metastases were detected by this assay in shVRK1-02 231-luc tumor bearing mice. (b) To assess the incidence and burden of metastases, peripheral tissues were excised from 231-Luc tumor-burdened mice at end point (13 weeks). Lysates were prepared from lymph nodes, liver, lungs and brain and luciferase activity was measured in vitro (RLU). Metastatic burden of seven individual mice from each cohort is represented by the dots, and the black bars indicate the average RLU \pm s.e. The statistical significance of tumor-derived luciferase expression in the tissues was assessed by the Mann-Whitney test $\left({ }^{* *} P<0.01,{ }^{* *} P<0.001\right)$.

depletion was maintained during the experiment; Supplementary Figure S1). These data provide strong evidence that VRK1 contributes directly to tumorigenesis.

VRK1 depletion reduces the incidence and burden of distal metastases

The identification of VRK1 as a marker of poor prognostic outcome in breast cancer patients, ${ }^{17}$ and that the observation that the C. elegans ortholog of VRK1 contributes to cellular invasion during development, ${ }^{26}$ led us to consider that VRK1 might have a role in metastasis as well as tumor growth. Lymph nodes, liver, lung and brain of tumor-burdened mice were harvested and the presence of luciferase-expressing metastatic cells was assessed ex vivo; metastatic incidence and burden are reported in Figure 7 and Table 1. Metastasis to the lymph nodes was frequent for
Table 1. Incidence of metastasis in tumor-burdened mice

\begin{tabular}{lcccc}
\hline Tumor & $\begin{array}{c}\text { Lymph } \\
\text { nodes }\end{array}$ & Liver & Lungs & Brain \\
\hline 231-Luc shSCRAM & $\begin{array}{l}4 / 7(57 \%) \\
\text { 231-Luc shVRK1-02 }\end{array}$ & $\begin{array}{l}2 / 7(71 \%) \\
1 / 7(14 \%)\end{array}$ & $\begin{array}{l}6 / 7(86 \%) \\
1 / 7(14 \%)\end{array}$ & $\begin{array}{l}2 / 7(29 \%) \\
0 / 7(0 \%)\end{array}$ \\
\hline
\end{tabular}

both 231-Luc shSCRAM and -shVRK1-02 tumor-burdened mice, while metastasis to the liver was less frequent but seen in both cohorts (Figure 7b, top). Breast cancer has a metastatic proclivity for the lungs, ${ }^{27}$ which was evident in the 231-Luc shSCRAM tumorburdened mice (Figure 7a), as well as for the brain. Mice engrafted with VRK1-depleted tumors had a significant reduction in both burden (Figure $7 \mathrm{~b}$ ) and incidence (Table 1) of lung metastases, 
and no brain metastases were seen in these mice. Cumulatively, our data indicate that VRK1 depletion not only reduces the size and rate of growth of primary tumors, but also diminishes distal metastasis to the brain and the lung.

\section{DISCUSSION}

The correlation of VRK1 overexpression with poor clinical outcome in a subset of breast cancer patients ${ }^{16,17}$ suggested that high levels of VRK1 might impact cell survival, proliferation and/or metastasis. Our studies were focused on human cell lines representing normal (MCF10a) and highly malignant (MDA-MB231) mammary epithelia, and are the first to address the role of the VRK1 protein kinase in these cells in vitro and in vivo.

We show that shRNA-mediated depletion of VRK1 in MCF10a cells slows their proliferation, causing an $11.5 \%$ (shVRK1-01) to $80 \%$ (shVRK1-02) increase in their apparent doubling time (Figure 1). Our data suggest a role for VRK1 in regulating the G1 $\rightarrow \mathrm{S}$ transition, which is consistent with what has been previously reported for WS1 primary fibroblasts. ${ }^{9}$ However, as we observed only a modest decrease in cyclin D levels upon VRK1 depletion (Figure 1), understanding the mechanisms whereby VRK1 accelerates passage through the G1/S restriction point is an important focus for future studies. For example, while the estrogen receptor is a putative target for VRK1, ${ }^{28}$ we do not know whether VRK1 (like VRK2) intersects with other mitogenic pathways. VRK1 was also found to have an important role in the proliferation of MDA-MB-231 cells, with their apparent doubling time increased by $\sim 40 \%$ by both shVRK1s (Figure 1). No shift in the cell cycle distribution of the population was seen (Figure 2), however, consistent with VRK1 having temporally distinct functions throughout the cell cycle.

Although an sixfold overexpression of VRK1 did not enhance MDA-MB-231 proliferation under normal growth conditions, a marked effect was seen under conditions of serum starvation. Relative to control cells, VRK1-overexpressing cells exhibited a reduced apoptotic response and a net increase in cell number (Figures $4 \mathrm{~b}$ and $\mathrm{c}$ ). Flow cytometric analysis confirmed that VRK1 overexpression led to modest but sustained proliferation (Figure 5), and the Pl-stained profiles of the two cell populations differed significantly. There was a marked leftward shift in the mean fluorescence intensity of the G0/G1 population in the control cells, consistent with either hypercompaction (reflecting exit into G0 and/or increased heterochromatinization) or loss of DNA. No such shift was seen in the VRK1-overexpressing cells, in which the transgene is unlikely to be responsive to endogenous physiological signals. Understanding how VRK1 is itself regulated by starvation or stress, and how its constitutive expression may override the induction of apoptosis or quiescence, is a key area of future study. In vivo, VRK1 overexpression may facilitate tumor cell survival under conditions of nutrient limitation before the induction of a proangiogenic program. Interestingly, overexpression of the membrane-bound VRK2 protein kinase, which has a high degree of sequence similarity to VRK1, can regulate the cellular response to hypoxia. ${ }^{29}$

Our studies on the role of VRK1 in cultured cells were complemented by our use of a murine orthotopic xenograft model of breast cancer (Figures 6 and 7). As assessed by both biophotonic imaging and caliper measurement, VRK1-depleted cells formed tumors that were consistently $\sim 50 \%$ smaller from 4 to 12 weeks postengraftment (Figure 6). An 18\% decrease $(P=0.063)$ was found in the proliferative index of the VRK1depleted tumors relative to control (Supplementary Figure S1), which is consistent with a direct impact on cell proliferation and supports the conclusion that VRK1 has a biologically relevant role in tumor growth in vivo.

Poor clinical outcome in breast cancer patients is usually associated with metastatic disease, which reflects intrinsic properties of the tumor cells, as well as the surrounding microenvironment. ${ }^{30-32}$ Significantly, our studies indicated that VRK1 depletion diminished or prevented metastasis to the brain or lungs (Figure 7). Although it is formally possible that this difference merely reflects the smaller size of the VRK1-depleted tumors, a direct role for VRK1 in cell invasion or colonization seems probable. In C. elegans, the VRK ortholog has been shown to have a role in cell invasion during development ${ }^{26}$ and VRK2 has recently been reported to affect cell invasiveness by regulating NFAT1 and modulating COX-2 expression. ${ }^{33}$ Direct examination of whether VRK1 can modulate MDA-MB-231 invasiveness in vitro will be another area of future interest.

In sum, our studies provide the first direct evidence that VRK1 affects the proliferation of mammary epithelial cells and the ability of such cells to survive nutrient limitation and avoid apoptosis or exit from the cell cycle. In addition, we show that VRK1 depletion causes a $50 \%$ decrease in the size of MDA-MB-231 tumors in mice, and prevents hematogenous metastasis to the brain or lungs. These data, coupled with previous reports documenting an association of VRK1 overexpression with poor clinical outcome in breast cancer patients, confirms VRK1 as a new candidate for chemotherapeutic intervention.

\section{MATERIALS AND METHODS}

\section{Cell culture}

Human mammary epithelial cell lines MCF10a (CRL-10317) and MDA-MB231 (HTB-26) were obtained from the ATCC (Rockville, MD, USA) and cultured as directed. Puromycin $(7.5 \mu \mathrm{g} / \mathrm{ml})$ or hygromycin $(50 \mu \mathrm{g} / \mathrm{ml})$ were added to the media of lentiviral-transduced cells, as appropriate. For serum starvation experiments, cells were refed every 3 days with media containing $0.2 \%$ serum and no additional growth factors or supplemental nutrition.

Lentivirus construction and transduction for short hairpin RNA (shRNA) expression

The pLL 3.7 LentiLox system (Addgene, Cambridge, MA, USA) was used to generate replication-deficient lentiviral particles for stable transduction of shRNA. The 'sense' strand of the shRNA sequences are as follows, with uppercase letters signifying the VRK1 target sequence. VRK1-01: $5^{\prime}$-tGA AAGAGAGTCCAGAAGTAttcaagagaTACTTCTGGACTCTCTTCtttttggaa ac-3', VRK1-02: sense, 5'-tGCAAGGAACCTGGTGTTGAttcaagagaTCAACACCAGGTT CCTTGCtttttggaaac-3', shSCRAM:5'-tCAGTCGCGTTGCGACTGGttcaagagaC CAGTCGCAAACGCGACTGtttttggaaac- $3^{\prime} .{ }^{34}$ Lentivirus stocks were prepared in 293 T cells; Puro transduced lines were used for experimental analysis from 4-25 days postinfection.

Lentiviral construction and transduction for transgene expression A variant of the pHAGE lentiviral system (generous gift of Dr Amy Hudson, Medical College of Wisconsin) was used to generate replication-deficient viral particles for stable transduction of complementary DNAs expressed

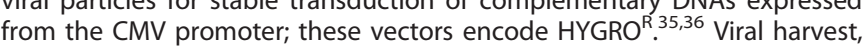
transduction and selection were performed as described for the Lentilox system.

Immunoblot analysis of cellular lysates

Cellular lysates were prepared in the presence of Benzonase Nuclease (Sigma-Aldrich, St Louis, MO, USA) and protease and phosphatase inhibitors; total protein concentration was determined using the BCA assay. Commercial antibodies used included: $\mathrm{Bcl}-\mathrm{xL}$ (CellSignaling, Danvers, MA, USA, 2762), cyclin D1 (SantaCruz, Dallas, TX, USA, SC20044), FLAG(M2) (Sigma-Aldrich, F3-165), PCNA (SantaCruz, sc-56), pP53-Thr18 (SantaCruz, SC-16716), pH3-S10 (Upstate, Billerica, MA, USA, 06-570), GAPDH (Imgenex, San Diego, CA, USA, 5019A-2), VRK1 (Sigma-Aldrich, HPA000660). The polyclonal anti-BAF antibody was generated commercially using amino acid 4-20 as the immunogen. Immunoblots were developed using the SuperSignal West Pico Chemiluminescence Substrate (Pierce, Rockford IL, USA) and quantitated on a FluorChemHD2 Imaging System (Protein Simple, Santa Clara, CA, USA). 
Determination of cell viability and apparent doubling time

Cells were seeded at a known density ( $n$ start) and grown for varying periods of time $(h)$, at which time the cell number ( $n$ final) was determined and the number of doublings that had occurred $(x)$ was calculated using the equation $2^{x}=n$ final $/ n$ start. The apparent doubling time $(h / x)$ was then calculated. The Millipore Guava ViaCount Assay system (Millipore, Billerica, MA, USA) was used for cell counting and to distinguishable viable versus dead cells during sequential passage.

\section{Quantification of apoptosis}

Spent medium was collected from individual culture dishes and assayed with the Caspase-Glo 3/7 Reagent (Promega, Madison, WI, USA) as directed. The relative light units (RLU), which correlates with caspase 3/7 activity Waltham, was measured on the Victor ${ }^{3} V$ Multilabel Plate Reader (Perkin-Elmer, Waltham, MA, USA). As controls for the assay, spent media were collected from cells cultured for $24 \mathrm{~h}$ under normal culture conditions in the presence or absence of a 6-h treatment with $1 \mu \mathrm{m}$ Staurosporine (Sigma-Aldrich). In addition, spent media were collected from MDA-MB231-PHM and MDA-MB-231-VRK1 cells after 7 days of culture under serum starvation conditions. In all cases, two biological replicates were performed; all samples were assayed in duplicate simultaneously. Significance was determined by $t$-test.

Analysis of cell cycle distribution by BrdU and propidium iodide staining

Flow cytometry was employed to analyze cell cycle dynamics using propidium iodide to assess relative DNA content and BrdU incorporation (1 $\mathrm{h}$ pulse) to monitor DNA synthesis. The FITC BrdU Flow Kit (BD Biosciences, San Jose, CA, USA) was used according to the manufacturer's specifications. Flow cytometry was performed on the Guava EasyCyte Miniflow cytometer; data were compiled using Guava ExpressPro software (Millipore) and analyzed using FlowJo software (TreeStar Inc., Ashland, OR, USA).

\section{Orthotopic xenograft and bioluminescence imaging}

MDA-MB-231 cells expressing the Firefly-Luciferase gene (Luc) ${ }^{25}$ were engineered to express shSCRAM or shVRK1-02 as described above. A total of $1.5 \times 10^{6}$ cells were injected into the 4th mammary fatpad of anesthetized female 8-10-weeks-old immune-deficient hairless-SCID mice (Crl:SHO-Prkdc ${ }^{\text {scid }} \mathrm{Hr}^{\text {hr }} \mathrm{SHO}^{\mathrm{TM}}$ mouse, Charles River, MA, USA). Following engraftment, mice were injected intraperitoneally with a $150 \mathrm{mg} / \mathrm{kg}$ dose of D-Luciferin (Caliper Life Sciences, Alameda, CA, USA). Bioluminescence was recorded on the Lumina-IVIS-100 In Vivo Imaging System (Xenogen Corp, Caliper Life Sciences) 5-10 min after injection and repeated until peak signal saturation was achieved. Regions of interest were assigned by the IVIS software based on signal intensity and reported as area flux, defined by radiance (photons $/ \mathrm{s} / \mathrm{cm}^{2} /$ steradian). Bioluminescence and caliper measurements of engrafted tumors were repeated weekly for 13 weeks; at end point, animals were injected intraperitoneally with $200 \mu \mathrm{l}$ of a $10 \mathrm{mg} / \mathrm{ml}$ solution of BrdU $48 \mathrm{~h}$ before being euthanized. Tumors and tissues were collected and snap frozen or fixed in $10 \%(\mathrm{v} / \mathrm{v})$ zinc formalin for biochemical or histological analyses.

\section{Detection of metastases by luciferase activity}

Tumor, lymph nodes (axillary, inguinal and mesenteric), liver, lungs and brain were excised from tumor bearing mice and homogenized in $1 \times$ Reporter Lysis Buffer (Promega) plus protease inhibitors. Thirty micrograms of protein of each sample was mixed with $100 \mu \mathrm{l}$ of Luciferase Assay Reagent (Promega); the luminescent signal was measured for $5 \mathrm{~s}$ on a Victor ${ }^{3} \mathrm{~V} 1420$ Multilabel Counter (Perkin-Elmer) and expressed as RLU/mg of total protein. Tissues from non-tumor bearing mice were assayed to determine the background inherent to the assay. Extracts exhibiting a signal of $>100$ RLU above background were rated positive for metastasis.

\section{CONFLICT OF INTEREST}

The authors declare no conflict of interest.

\section{ACKNOWLEDGEMENTS}

We thank Dr Michael Dwinell (MCW) for the gift of the MDA-MB-231-Luc cells and guidance with the orthotopic xenograft model, Dr Stephen Duncan (MCW) for sharing the LentiLox system, Dr Amy Hudson (MCW) for sharing the modified pHAGE system, Drs Jeremy Nichols and Matthew Wiebe (MCW) for help with establishing the shRNA system and Dr Kathleen Boyle for her comments on the manuscript. We also acknowledge Dr Alexandra Lerch-Gaggl and the Analytical Tissue Core facility for assistance with histological analysis. This work was supported in part by generous funds from the Advancing a Healthier Wisconsin initiative and the Wisconsin Breast Cancer Showhouse funding program.

\section{REFERENCES}

1 Hanahan D, Weinberg RA. Hallmarks of cancer: the next generation. Cell 2011; 144: 646-674.

2 Nichols RJ, Traktman P. Characterization of three paralogous members of the Mammalian vaccinia related kinase family. J Biol Chem 2004; 279: 7934-7946.

3 Nezu J, Oku A, Jones MH, Shimane M. Identification of two novel human putative serine/threonine kinases, VRK1 and VRK2, with structural similarity to vaccinia virus B1R kinase. Genomics 1997; 45: 327-331.

4 Vega FM, Gonzalo P, Gaspar ML, Lazo PA. Expression of the VRK (vaccinia-related kinase) gene family of p53 regulators in murine hematopoietic development. FEBS Lett 2003; 544: 176-180.

5 Santos CR, Rodriguez-Pinilla M, Vega FM, Rodriguez-Peralto JL, Blanco S, Sevilla $A$ et al. VRK1 signaling pathway in the context of the proliferation phenotype in head and neck squamous cell carcinoma. Mol Cancer Res 2006; 4: 177-185.

6 Valbuena A, Lopez-Sanchez I, Vega FM, Sevilla A, Sanz-Garcia M, Blanco S et al. Identification of a dominant epitope in human vaccinia-related kinase 1 (VRK1) and detection of different intracellular subpopulations. Arch Biochem Biophys 2007; 465: 219-226.

7 Vega FM, Sevilla A, Lazo PA. p53 Stabilization and accumulation induced by human vaccinia-related kinase 1. Mol Cell Biol 2004; 24: 10366-10380.

8 Kang TH, Park DY, Kim W, Kim KT. VRK1 phosphorylates CREB and mediates CCND1 expression. J Cell Sci 2008; 121(Pt 18): 3035-3041.

9 Valbuena A, Lopez-Sanchez I, Lazo PA. Human VRK1 is an early response gene and its loss causes a block in cell cycle progression. PLOS ONE 2008; 3: e1642.

10 Wiebe MS, Nichols RJ, Molitor TP, Lindgren JK, Traktman P. Mice deficient in the serine/threonine protein kinase VRK1 are infertile due to a progressive loss of spermatogonia. Biol Reprod 2010; 82: 182-193.

11 Choi YH, Park CH, Kim W, Ling H, Kang A, Chang MW et al. Vaccinia-related kinase 1 is required for the maintenance of undifferentiated spermatogonia in mouse male germ cells. PLOS ONE 2010; 5: e15254.

12 Schober CS, Aydiner F, Booth CJ, Seli E, Reinke V. The kinase VRK1 is required for normal meiotic progression in mammalian oogenesis. Mech Dev 2011; 128: 178-190.

13 Gorjanacz M, Klerkx EP, Galy V, Santarella R, Lopez-Iglesias C, Askjaer P et al. Caenorhabditis elegans BAF-1 and its kinase VRK-1 participate directly in postmitotic nuclear envelope assembly. EMBO J 2007; 26: 132-143.

14 Nichols RJ, Wiebe MS, Traktman P. The vaccinia-related kinases phosphorylate the $\mathrm{N}^{\prime}$ terminus of BAF, regulating its interaction with DNA and its retention in the nucleus. Mol Biol Cell 2006; 17: 2451-2464.

15 Kang TH, Park DY, Choi YH, Kim KJ, Yoon HS, Kim KT. Mitotic histone H3 phosphorylation by vaccinia-related kinase 1 in mammalian cells. Mol Cell Biol 2007; 27: 8533-8546.

16 Fournier MV, Martin KJ, Kenny PA, Xhaja K, Bosch I, Yaswen P et al. Gene expression signature in organized and growth-arrested mammary acini predicts good outcome in breast cancer. Cancer Res 2006; 66: 7095-7102.

17 Martin KJ, Patrick DR, Bissell MJ, Fournier MV. Prognostic breast cancer signature identified from 3D culture model accurately predicts clinical outcome across independent datasets. PLOS ONE 2008; 3: e2994.

18 Valbuena A, Sanz-Garcia M, Lopez-Sanchez I, Vega FM, Lazo PA. Roles of VRK1 as a new player in the control of biological processes required for cell division. Cell Signal 2011; 23: 1267-1272.

19 Barcia R, Lopez-Borges S, Vega FM, Lazo PA. Kinetic properties of p53 phosphorylation by the human vaccinia-related kinase 1. Arch Biochem Biophys 2002; 399: 1-5.

20 Lopez-Borges S, Lazo PA. The human vaccinia-related kinase 1 (VRK1) phosphorylates threonine- 18 within the $\mathrm{mdm}-2$ binding site of the $\mathrm{p} 53$ tumour suppressor protein. Oncogene 2000; 19: 3656-3664.

21 Hui L, Zheng Y, Yan Y, Bargonetti J, Foster DA. Mutant p53 in MDA-MB-231 breast cancer cells is stabilized by elevated phospholipase $D$ activity and contributes to survival signals generated by phospholipase D. Oncogene 2006; 25: 7305-7310.

22 Hanahan D, Weinberg RA. The hallmarks of cancer. Cell 2000; 100: 57-70. 
23 Valenti F, Fausti F, Biagioni F, Shay T, Fontemaggi G, Domany E et al. Mutant p53 oncogenic functions are sustained by Plk2 kinase through an autoregulatory feedback loop. Cell Cycle 2011; 10: 4330-4340.

24 Hainaut P, Hollstein M. P53 and Human Cancer: the first ten thousand mutations. Adv Cancer Res 2000; 77: 81-137.

25 Wendt MK, Cooper AN, Dwinell MB. Epigenetic silencing of CXCL12 increases the metastatic potential of mammary carcinoma cells. Oncogene 2008; 27: 1461-1471.

26 Klerkx EP, Alarcon P, Waters K, Reinke V, Sternberg PW, Askjaer P. Protein kinase VRK-1 regulates cell invasion and EGL-17/FGF signaling in Caenorhabditis elegans. Dev Biol 2009; 335: 12-21.

27 Minn AJ, Gupta GP, Siegel PM, Bos PD, Shu W, Giri DD et al. Genes that mediate breast cancer metastasis to lung. Nature 2005; 436: 518-524.

28 Sanz-Garcia M, Vazquez-Cedeira M, Kellerman E, Renbaum P, Levy-Lahad E, Lazo PA. Substrate profiling of human vaccinia-related kinases identifies coilin, a Cajal body nuclear protein, as a phosphorylation target with neurological implications. J Proteomics 2011; 75: 548-560.

29 Blanco S, Santos C, Lazo PA. Vaccinia-related kinase 2 (VRK2) modulates the stress response to hypoxia mediated by TAK1. Mol Cell Biol 2007; 27: 7273-7283.

30 Mbeunkui F, Johann Jr. DJ. Cancer and the tumor microenvironment: a review of an essential relationship. Cancer Chemother Pharmacol 2009; 63: 571-582.
31 van 't Veer $L J$, Dai $H$, van de Vijver MJ, He YD, Hart AA, Mao M et al. Gene expression profiling predicts clinical outcome of breast cancer. Nature 2002; 415 : 530-536.

32 Weigelt B, Peterse JL, van 't Veer $\mathrm{U}$. Breast cancer metastasis: markers and models. Nat Rev Cancer 2005; 5: 591-602.

33 Vazquez-Cedeira M, Lazo PA. Human VRK2 (Vaccinia-related Kinase 2) Modulates Tumor Cell Invasion by Hyperactivation of NFAT1 and Expression of Cyclooxygenase-2. J Biol Chem 2012; 287: 42739-42750.

34 Jacque JM, Stevenson M. The inner-nuclear-envelope protein emerin regulates HIV-1 infectivity. Nature 2006; 441: 641-645.

35 Schneider CL, Hudson AW. The human herpesvirus-7 (HHV-7) U21 immunoevasin subverts NK-mediated cytoxicity through modulation of MICA and MICB. PLOS Pathog 2011; 7: e1002362.

36 Mostoslavsky G, Fabian AJ, Rooney S, Alt FW, Mulligan RC. Complete correction of murine Artemis immunodeficiency by lentiviral vector-mediated gene transfer. Proc Natl Acad Sci USA 2006; 103: 16406-16411.

Oncogenesis is an open-access journal published by Nature Publishing Group. This work is licensed under a Creative Commons AttributionNonCommercial-NoDerivs 3.0 Unported License. To view a copy of this license, visit http://creativecommons.org/licenses/by-nc-nd/3.0/

Supplementary Information accompanies this paper on the Oncogenesis website (http://www.nature.com/oncsis). 